\title{
Nivel de cumplimiento de la Norma Oficial Mexicana 168-SSA1-1998. Una perspectiva del profesional de Enfermería
}

\author{
Level of fulfillment of the Mexican Health Standard \\ NOM 168-SSA1-1998. \\ A perspective from the Nursing Professional. \\ M.E. Sandra Hernández Corral• \\ L.E.O Martha Patricia Vargas Álvarez•• \\ L.E.O Alberto Domínguez Aliphat... \\ Mtra. Reyna Matus Miranda....
}

\section{Resumen}

Introducción: Los registros que realiza el profesional de enfermería son el soporte de los cuidados que brinda a cada una de las personas que le son asignadas en su jornada laboral, además son un elemento primordial para la toma de decisiones en la gestión del cuidado; por lo tanto es importante que los registros se realicen dando cumplimiento a la NOM 168-SSA1-1998 del expediente clínico para favorecer la continuidad y seguridad del cuidado; por lo cual el objetivo del estudio fue comparar el nivel de cumplimiento de la NOM 168-SSA1.1998 del expediente clínico en dos Institutos Nacionales de Salud, desde la perspectiva del profesional de enfermería. Material y métodos: Se realizó un estudio transversal, observacional, descriptivo y comparativo, la muestra estuvo integrada por 186 enfermeras. El nivel de cumplimiento de la NOM 168-SSA1-1998 se midió con una escala tipo likert modificado con 5 opciones de respuesta; para el análisis de datos se utilizó estadística descriptiva e inferencial. Resultados: Los datos obtenidos mostraron que la mayoría de las enfermeras refieren utilizar en sus registros lenguaje técnico, abreviaturas universales y letra legible, evitan realizar tachaduras y enmendaduras. Se observaron diferencias entre los dos institutos con relación al nivel de cumplimiento de la NOM 168-SSA1-1998 con un valor de $p<0.05$. Conclusiones: Desde el punto de vista de las enfermeras el nivel de cumplimiento en la norma es adecuado lo cual puede limitar su disposición para participar en programas de mejora.

\section{Palabras \\ Clave:}

Enfermería, registros

clínicos, nivel de

cumplimiento.

- Maestra en Enfermería, Subjefe de Educación e Investigación en Enfermería del Instituto Nacional de Rehabilitación •-Licenciada en Enfermería y Obstetricia, Subjefe de Educación e Investigación en Enfermería del Instituto Nacional de Cancerología ...Licenciado en Enfermería y Obstetricia, Jefe de terapia Intensiva del Instituto Nacional de Cancerología ...Maestra en Educación, Jefe de la División de Estudios de Posgrado ENEO Sandra Hernández Corral Correo electrónico: shcorral@gmail.com

RECIBIDO: 14 ENERO 2011 ENVIADO: 8 FEBRERO 2011 ACEPTADO: 3 MARZO 2011 


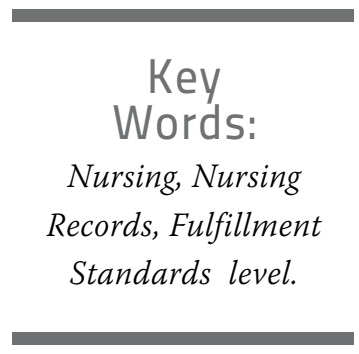

\begin{abstract}
Introduction: Mexican nursing professionals back up their care interventions through registers, and this information becomes fundamental in the process of decision-making. Therefore, in order to promote care continuity and security, it is important that these registers fulfill the Mexican health standard NOM 168SSA 1-1998. The objective of this study was to compare the levels of fulfillment of this standard in two National Institutes of Health. Methods and material: A basic, observational, and comparative study was conducted with a sample of 186 nurses. The level of standard fulfillment was estimated using a 5 point Likert Scale. Descriptive and inferential statistics were performed. Results: our findings suggest that the majority of nurses use technical language with legible and neat writing. A difference was found between the two institutes ( $p<$ .05). Conclusions: from the perspective of these nurses, the level of fulfillment of this standard was adequate.
\end{abstract}

\section{INTRODUCCION}

Los registros presentes en las hojas de enfermería son el soporte de los cuidados que la enfermera brinda a cada una de las personas que les son asignadas en su jornada laboral, son un elemento importante para la toma de decisiones durante la gestión del cuidado, convirtiéndose así en un registro esencial de gran valor legal e indicador de calidad, que al cubrir ciertos requisitos favorecen la continuidad y seguridad de la atención. ${ }^{1}$ Estos registros son considerados como una herramienta imprescindible para la comunicación, intra e interdisciplinar que hacen posible el intercambio de información sobre los cuidados permitiendo diferenciar la actuación de enfermería de la de otros profesionales de la salud. ${ }^{2}$

Los registros de enfermería constituyen un instrumento de gestión que respalda el quehacer profesional de la enfermera; son una parte fundamental de la asistencia sanitaria y están integrados en el expediente clínico del paciente, por tanto están sujetos a la normatividad establecida, que en caso de no cumplirse conlleva una serie de repercusiones y responsabilidades de índole profesional y legal. En México ésta normatividad está dada por la Norma Oficial Mexicana NOM-168-SSA1-1998 del Expediente clínico que en relación a la hoja de enfermería puntualiza lo siguiente. "Deberá elaborarse por el personal de turno, según la frecuencia establecida por las normas del establecimiento y las órdenes del médico y deberá contener como mínimo: habitus exterior, gráfica de signos vitales, ministración de medicamentos, fecha, hora, cantidad y vía, procedimientos realizados y observaciones."
De ahí la importancia que el profesional de enfermería conozca, efectivamente, esta reglamentación.

La Comisión Nacional de Arbitraje Médico al considerar la importancia de los registros clínicos de enfermería como una evidencia, emite las siguientes recomendaciones: Establecer una coordinación efectiva con el equipo interdisciplinario de salud aplicando a cada uno de los documentos (expediente clínico, reportes, informes etc.) los lineamientos establecidos por la institución, realizarlos en forma clara, legible, veraz, oportuna y confiable, utilizando terminología técnico-médica de uso y aceptación universal. ${ }^{4}$

Los registros de enfermería se han estudiado desde diferentes vertientes, tales como la trascendencia, la calidad, la utilización del proce- 
so de enfermería, la comparación de diferentes métodos de registros, la calidad del cuidado y propuestas encaminadas a mejorar esos registros. En 1999 se realizó un estudio en relación a la trascendencia que tienen los registros de enfermería en el expediente clínico de los pacientes hospitalizados en el servicio de Urgencias y Unidad coronaria del Instituto Nacional de Cardiología "Ignacio Chávez" donde se reportó que un $80.6 \%$ de su personal desconocía las normas elaboradas para los registros de enfermería; el estudio concluye que el personal cuenta con escasa experiencia en el manejo de los registros de enfermería lo cual trae como consecuencia que sean deficientes. ${ }^{5}$

Al investigar la calidad de los registros de enfermería, algunos autores reportan que dentro de las principales anormalidades encontradas tienen que ver con que éstas son realizadas por turno y no por horario, que presentan borrones, tachaduras y espacios en blanco o ausencia de la firma. Otros autores han encontrado que estos registros se hacen de manera general y aunque fueron comprensibles, en ocasiones la letra fue poco legible, ya que se utilizaron siglas estandarizadas y términos técnicos; ${ }^{6}$ sin embargo hay investigaciones que destacan que las enfermeras al elaborar sus registros utilizan solo términos generalizados, evasivos y de abreviaturas que dificultan la comprensión de registros. ${ }^{7,8}$

Entre los conceptos que el personal de enfermería aplica en sus registros: se encuentran los datos de identificación observándose que todos los registros presentaron el nombre del paciente, algunos otros elementos presentaron altos por- centajes de cumplimiento como: fecha, número de cama, servicio y diagnóstico. En el rubro correspondiente a la firma de la enfermera, solamente se observó en un $79.4 \%$, situación que es de llamar la atención ya que el personal de enfermería es quien realiza los registros. ${ }^{9}$

Existen investigaciones que se han centrado en evaluar la documentación de los registros de enfermería antes y después de la implementación de un sistema de registro electrónico; mientras que en algunos se reporta que después de implementar el sistema para la documentación, la atención del paciente es más precisa, completa y oportuna, otros no reportan diferencias de la documentación de enfermería después de la implementación. En algunos reportes las enfermeras consideran el sistema informático como una mejora del método de trabajo, frente al sistema de bolígrafo-papel. Estos resultados se asocian con los factores de tipo organizacional donde el tipo y la duración de la capacitación juegan un papel importante. Ante estos hallazgos existen autores que destacan la necesidad de evaluar la documentación de enfermería después de la introducción de un sistema electrónico y no considerar que por el simple hecho de utilizarlo la información que se documenta sea completa y precisa para la toma de decisiones. ${ }^{10-13}$

Con el fin de aumentar la calidad de los registros de enfermería, se han creado propuestas que manifiestan la necesidad de contar con instrumentos de valoración inicial que favorezcan la continuidad de la atención, a través de un cumplimiento riguroso del registro del cuidado proporcionado, así como de las estrategias de comunicación inter o intradisciplinar uti-

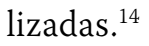

Otra de las estrategias empleadas para detectar oportunidades de mejora y proponer medidas correctivas para el sistema de registros de enfermería, son los indicadores de calidad, utilizados frecuentemente en las auditorias de proceso. Por ejemplo en España al realizar una auditoría, en una unidad de hemodiálisis, se reportaron inconsistencias en los documentos utilizados para el registro de enfermería, entre las que se encontraron por un lado, que las actividades de cuidado no se veían reflejadas o eran escasas, por otro lado que las anotaciones realizadas eran difíciles de leer por sus colegas; así mismo encontraron que el conocimiento de la norma era sólo parcial y que las implicaciones legales eran un factor determinante al realizar el registro. ${ }^{15}$

Al tomar en cuenta el Proceso Enfermero como punto de referencia para la elaboración de los registros clínicos, se ha observado una variabilidad, puesto que hay estudios que excluyen la etapa del Diagnóstico enfermero, o sólo incluyen la fase de ejecución como parte del registro, incluso algunos toman en cuenta todo el proceso pero no se realiza de forma completa. En cuanto a la percepción de la utilidad del proceso de enfermería para elaborar los registros clínicos, las enfermeras opinan que les traerá algún beneficio y que la información recabada de los pacientes a su cargo será mayor, opinan que la asistencia a los pacientes mejorará con la aplicación del proceso, y un menor porcentaje tienen la certeza que su aplicación les dará mayor reconoci- 
miento profesional; sin embargo algunas consideran que la aplicación del proceso, únicamente les traerá mayor carga de trabajo. ${ }^{16-19}$

Como se puede apreciar, la investigación de los registros clínicos de enfermería se han abordado desde diferentes perspectivas, sin embargo poco se ha estudiado acerca del apego a las normas establecidas para elaborarlos. Esto resulta de vital importancia, puesto que los profesionales de enfermería deben conocer y llevar a la práctica la legislación vigente, a fin de evitar las implicaciones jurídicas que el no hacerlo de esta forma, conlleva.

Considerando lo anterior es importante analizar el nivel de cumplimiento de la NOM 168SSA1.1998 del expediente clínico en dos Institutos Nacionales de Salud, desde la perspectiva del profesional de enfermería, lo cual permitirá proponer estrategias dirigidas a mejorar la calidad de los registros.

\section{Metodología}

El estudio fue transversal, observacional y comparativo. El universo fue de 897 enfermeras (Instituto 1: 467, Instituto 2: 430), la población de estudio fue de 129 del Instituto 1 y 115 del Instituto 2, todas ellas de los servicios de hospitalización. La muestra fue no aleatoria por conveniencia y se calculo utilizando la fórmula para poblaciones finitas, con un nivel de confianza del 95\%, quedando integrada por 97 enfermeras del instituto 1 y 89 del instituto 2 .

Criterios de inclusión: todas las enfermeras que realizan registros clínicos en los servicios de hospitalización, que estuvieron presentes en el momento de aplicar el instrumento y que aceptaron responder; se excluyeron a las enfermeras que no aceptaron participar en el estudio.

La variable de estudio fue el nivel de cumplimiento de los registros clínicos de enfermería; ésta se midió con un instrumento que contempla seis dimensiones: Identificación con seis indicadores con un valor de 30 puntos; Habitus exterior con 2 indicadores y un valor de 10 puntos; Signos vitales con 4 indicadores y un valor de 20 puntos; Ministración de medicamentos con 9 indicadores con valor de 45 puntos; Cuidados de enfermería con 7 indicadores y 35 puntos y Observaciones con 2 indicadores y 10 puntos. El nivel de cumplimiento tiene una puntuación que va de 30 a 150 puntos en donde se consideró los siguientes puntos de corte: de 30 a 70 Inadecuado, de 71 a 110 moderadamente adecuado y de 111 a 150 adecuado.

La recolección de los datos fue a través de un instrumento diseñado exprofeso por los autores retomando la NOM-168-SSA1-1998 del expediente clínico; este fue sometido a validez de contenido por un grupo de expertos de la Comisión Científica de Investigación en Enfermería Oncológica del INCan. El instrumento consistió en un cuestionario de autoaplicación, con 41 preguntas tipo Likert modificado con opciones de respuesta de nunca (1) a siempre (5), de las cuales cinco corresponden a variables sociodemográficas, seis a las características de los registros y treinta al nivel de cumplimiento de la norma. Se sometió a una prueba piloto alcanzando un índice de confiabilidad de Alfa de Cronbach de 0.86 .

Se construyó una base de datos utilizando el paquete estadístico
SPSS Versión 18 y para realizar el análisis se utilizaron medidas de tendencia central y estadística inferencial utilizando la prueba $\mathrm{T}$ de Student para muestras independientes.

Se solicitó la colaboración de las enfermeras de forma verbal, respetando ante todo la autonomía y confidencialidad de la información que proporcionaron los participantes.

\section{Resultados}

Se estudiaron un total de 186 profesionales de enfermería, de los cuales el 88.7\% fueron mujeres, el grupo de edad con mayor porcentaje fue el de 31 a 40 años con el 61.3\%, seguido del grupo de 21 a 30 años con el 26.9\%; el 34.4\% labora en el matutino, el $30.06 \%$ en el vespertino y el 35\% en el nocturno. La antigüedad laboral para el grupo de $0 \mathrm{a}$ 10 años fue del $63.4 \%$ y $30.6 \%$ en el de 11 a 20 años. Respecto al grado académico, el 40.3\% tienen licenciatura, el $25.3 \%$ son de nivel técnico, el $18.8 \%$ tienen curso postécnico y 15.6\% estudios de posgrado.

Con respecto a las características de los registros clínicos que el personal de enfermería maneja, los promedios obtenidos fueron: respecto a la terminología médica (4.39), al uso de abreviaturas universales (3.92), letra legible (4.63), hoja de registros clínicos en buen estado (4.85), y evitar realizar enmendaduras (4.34) y tachaduras (4.47).

A continuación se describen los promedios por dimensiones evaluadas, los resultados fueron: Identificación 29.08, Habitus exterior 9.05, Signos vitales 19.44, Ministración de medicamentos 42.96, Procedimientos realizados 31.88 y Observaciones 8.99. El nivel de cumplimiento de la NOM-168-SSA1-1998 del 
expediente clínico en relación con los registros clínicos elaborados por las enfermeras de dos institutos fue de 141.17 lo que corresponde a un nivel adecuado.

Al comparar el nivel de cumplimiento de la Norma en ambos Institutos, se encontraron diferencias entre ellos ( $t=3.979, \mathrm{gl}=184 \mathrm{y}$ $\mathrm{p}=0.000$ ). Como puede observarse en la gráfica 1.

En la Tabla 1 se pueden apreciar los resultados de la comparación del nivel de cumplimiento por dimensiones de la NOM-168SSA1-1998 del expediente clínico de dos Institutos Nacionales de Salud. Las diferencias existentes son en las dimensiones de Ministración de medicamentos, cuidados de enfermería y observaciones, se aprecia que los promedios más altos corresponden al instituto 1.

\section{Discusıón}

Las características de los registros de enfermería referidos alcanzaron promedios altos con relación al uso de terminología médica, abreviaturas autorizadas, letra legible y mantener la hoja de registros clínicos en buen estado, también mencionan evitar realizar enmendaduras y tachaduras, estos resultados son similares a lo reportado por Vargas-Cubero, ${ }^{8}$ quien reporta que las enfermeras en su mayoría usan letra legible y abreviaturas autorizadas, además de mantener los registros pulcros; sin embargo, nuestros resultados difieren a los obtenidos por Torruella-Farre en el

\section{GRAFICA 1}

Comparación del nivel de cumplimiento de la NOM-168-SSA1-1998 del expediente clínico en dos Institutos Nacionales de salud

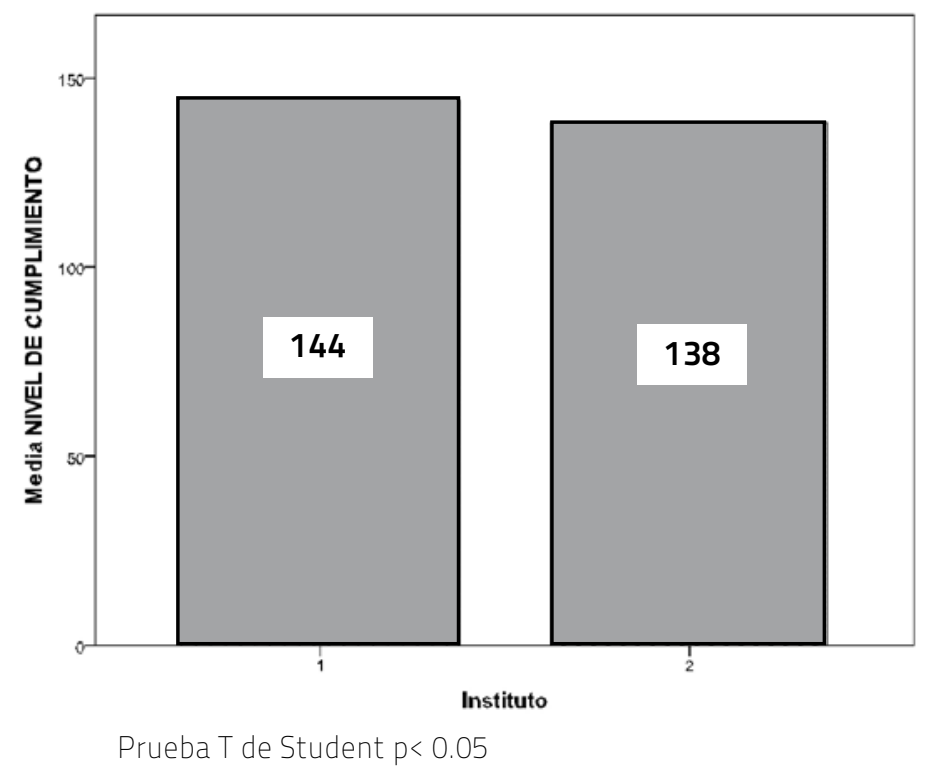

\section{Tabla 1. Comparación de las dimensiones de la NOM-168-SSA1-1998 del expediente clínico en dos Institutos Nacionales de salud}

\begin{tabular}{lrrr} 
DIMENSIONES & $\begin{array}{r}\text { Instituto 1 } \\
\mathrm{n}=97\end{array}$ & $\begin{array}{r}\text { Instituto 2 } \\
\mathrm{n}=89\end{array}$ & Valor de p \\
\hline Identificación (30) & 29.11 & 28.89 & .613 \\
Habitus exterior (10) & 9.22 & 8.92 & .167 \\
Signos vitales (20) & 19.23 & 19.65 & .194 \\
Ministración de medicamentos (45) & 43.90 & 41.87 & .000 \\
Procedimientos realizados (35) & 33.12 & 30.54 & .000 \\
Observaciones (10) & 9.51 & 8.40 & .000
\end{tabular}


$2009^{20}$, que después de revisar 100 registros de enfermería encontró el uso frecuente de abreviaturas no autorizadas p.ej. iniciales y/o símbolos, registros ilegibles lo que hizo imposible identificar al profesional de enfermería que los realizó. Contrario al estudio realizado por $\mathrm{Vi}$ lleda-García, ${ }^{21}$ el personal observado refiere ausencia de tachaduras y enmendaduras tanto en el Instituto 1 como en el Instituto 2. Lo mismo sucede con el uso de términos generalizados/evasivos y de siglas o abreviaturas que dificultan la comprensión de los registros.?

La identificación correcta del paciente es una de las metas internacionales de la seguridad del paciente, por lo cual es necesario que se realice de acuerdo con las normas internacionales establecidas, pero tomando en cuenta el contexto específico de cada institución; en este sentido, el personal entrevistado afirma que casi siempre registran esta información, hecho que coincide con lo reportado por Tovar ${ }^{9}$ quien encontró los datos de identificación en todos los registros, pero que contrasta con lo descrito por Pokorski ${ }^{16}$ y colaboradores quienes comentan que el nombre del paciente estaba ausente en casi la mitad de los registros de una unidad quirúrgica.

Con respecto al Habitus exterior se encontró que las enfermeras comentan que casi siempre realizan esta valoración, este hecho coincide con Pokorski y colaboradores que observaron que más de la mitad de los registros la contenían. El promedio de cumplimiento encontrado fue alto en comparación con lo reportado por Tovar quien encontró un porcentaje mínimo de cumplimiento
Con relación a los signos vitales las enfermeras afirman que siempre los registran, estos resultados son congruentes con los obtenidos en el estudio titulado Repercusión de un programa de mejora continua en los registros de enfermería en el que se reporta que la curva de signos vitales se realiza en un alto porcentaje. ${ }^{22}$ Situación que es de esperarse puesto que forma parte de una práctica en el proceso de atención del paciente y que se realiza, en la mayoría de las veces, casi en forma automática.

Así mismo en la administración de medicamentos casi siempre la enfermeras registran nombre del medicamento, dosis, fecha y hora de forma correcta, esto coincide a lo reportado en un estudio realizado en una sala de neonatología, donde los niveles de eficiencia en el proceso de medicación fueron superiores al 90\%; sin embargo otro estudio reporta que el 12\% de registros no incluyen la vía de ministración de medicamentos, omisión que repercute directamente en la seguridad del paciente.

Los procedimientos realizados son equivalentes al plan de cuidados y con relación a su registro las enfermeras comentaron que casi siempre lo llevan a cabo, este hecho concuerda con el estudio titulado proceso de enfermería de la literatura a la práctica donde se menciona que los pacientes tenían un plan de cuidados con mayor frecuencia en las unidades clínicas y más completa en la terapia intensiva.

Finalmente, con relación al aspecto de observaciones las enfermeras mencionan que casi siempre lo realizan, estos resultados son similares con lo reportado por Vargas-Cubero que observó en un alto porcentaje de los registros había evidencia de la evolución de los pacientes.

El nivel de cumplimiento de la NOM 168-SSA1-1998 manifestado por los profesionales de enfermería fue adecuado, sin embargo los resultados difieren de los obtenidos por Villeda-García en un estudio llevado a cabo en un Instituto Nacional de Salud el cual calificó como de regular el cumplimiento de la Norma en la mitad de los registros y en un mínimo porcentaje como inadecuado.

Como se puede apreciar la NOM-168-SSA1-1998 del expediente clínico establece los parámetros que deben registrarse en la hoja de enfermería y que son de carácter obligatorio, su cumplimiento es imprescindible para la seguridad del paciente.

En este estudio se presenta la perspectiva de los profesionales de enfermería, respecto al cumplimiento de la normatividad establecida para los registros clínicos, la información obtenida permitirá a los responsables de la gestión del cuidado crear estrategias para mejorar dichos registros, lo que implica un reto mayúsculo, ya que además de considerar los resultados de las auditorías de registros clínicos también tendrá que tomar en cuenta que los profesionales están convencidos de cumplir con la normas establecidas y no perciben como una necesidad mejorar sus registros.

\section{Nota:}

Este estudio fue realizado como parte del programa de trabajo 20102011 de la Red de Unidades de Investigación en Enfermería ENEOUNAM Instituciones e Institutos Nacionales de Salud 


\section{Referencias Bibliográficas}

1 Secretaría de Salud. Decálogo del código de ética para las enfermeras y enfermeros de México. 2001. México. Disponible en: http://bit. ly/vLPyhf [Consulta 19 de mayo 2010]

2 García SR, Navío MA. Valentín ML. Normas básicas para la elaboración de los registros de enfermería. Nure Investigación. 2007; 28, Mayo-Junio Disponible en: http:// bit.ly/s5]470 [Consulta 29 de mayo 2010]

3 Norma Oficial Mexicana Del expediente Clínico NOM-168SSA1-1998. Disponible en: http:// bit.ly/bbDhhe [Consulta el 15 de abril 2010]

4 Comisión Nacional de Arbitraje Médico. Recomendaciones para mejorar la atención de enfermería. 2003. [En red]. Disponible en: http//www.conamed.gob.mx [Consulta 15 de mayo 2010]

5 Méndez MC, Morales CM, Preciado LM, Ramírez NM. La trascendencia que tienen los registros de enfermería en el expediente clínico. Revista Mexicana de Enfermería Cardiológica 2001; 9 (1-4): 11-7.

6 Luz A. Martins AP, Dynewicz AM. Características de las anotaciones encontradas en auditoría. Rev, Electr. Enf. [Internet]. 2007; 9 (2): 344-61. Disponible en: http://bit. ly/sQiRwR [Consulta 15 de mayo 2010]

7 Matsuda LM, Silva DMP, Evora YDM, Coimbra JAH. Apuntes/registros de enfermería: instrumento de comunicación para la calidad del cuidado. Rev, Electr. Enf. [Internet]. 2006; 8 (3): 415-21. Disponible en: http://bit.ly/tRrY7X [Consulta 17 de mayo 2010]

8 Vargas-Cubero C, Ruíz Sánchez MA. Aspectos legales de las notas de
Enfermería. Enfermería en Costa Rica. 2003;25 (1): 11-8.

9 Tovar LB. Correspondencia conceptual entre los registros de enfermería y el modelo de Virginia Henderson. Desarrollo Científ Enferm. 2004; 12 (5): 137-14.

10 Larrabe JH, Baldereghini S, ElderSorrelies K, Turner Z, Wender $R$, Hart J, Lenzi PS. Evaluation of documentation before and after implementation of a nursing information system in acute care hospital. Computers, Informatics, Nursing, 2001; 19(3): 56- 65.

11 Murphy Ch, Maynard M, Morgan G. Pretest and postest attitudes of nursing personnel toward a patient care information system. Computers in Nursing. 1994; 12 (5): 23944.

12 Alegría-Capel A, Ruíz-Lopez FJ, Hernández-Pérez F, Alemán-Alemán M, Barcia-Planes MA, Bernal-Valverde I. Registros de Enfermería en UCI. Enfermería Global. [Internet]. 2003 (2). Disponible en: http://www.um.es/eglobal/ [Consulta 23 mayo 2011]

13 González SJ, Cosgaya OG, Simón MJ. Bles AIM. Registros de Enfermería: convencional frente a informatizado. Unidad de Cuidados Críticos. Enferm. Intensiva. 2004; 15 (2): 53-62.

14. Palacios GR, Bejuca MA, Bejuca MM, Sálces SE. De la teoría a la práctica: registros para el cuidado del paciente renal. Enfermería Global [Internet]. 2004; Disponible en: http://www.um.es/eglobal/ [Consulta 29 de junio 2010]

15 Hernández D, Rodríguez CM, Gutiérrez NM, Bolaños HG. Elaboración de un registro enfermero para hemodiálisis según la norma ISO 9001:2005. Rev Soc Esp Enferm Nefrol 2007; 10 (1): 15-22.
16 Pokorski S, Moraes M, Chiarelli R, Paganini A, Rejane E. Proceso de enfermería: de la literatura a la práctica: ¿Qué estamos haciendo?. Rev Latin-am Enfermagem [Internet]. 200917 (3). Disponible en: http://www.eerp.usp.br/rlse [Consultado 27 mayo 2010]

17 Higuchi KA, Dulberg C, Duff V. Factors associated with nursing diagnosis utilization in Canada. Nurs Diagn 1999; 10 (4):137-47.

18 Repetto MA, Souza MF, Avaliaçāo da relalizaçāo e do registro da sistematizaçāo da assitência de enfermagem (SAE) em um hospital universitario. Rev Bras Enfermagem 2005; 58 (3): 325-29.

19 Loza BH, Vasquez VM. Impacto de la implementación del "Proceso de Atención de Enfermería" en el INNN MVS, mediante una prueba piloto. Enf. Neurol. (Mex).2005; 4 (1): 1-6.

20 Tarruella-Farre M. Hojas de registros clínicos de enfermería; cumpliendo con los requisitos legales. Rev Enferm, 2009 Oct;32 (10): 54-58

21 Villeda-García FL, Cordova-Avila MA, Rodríguez-Suárez, BalbuenaRomán S, Hernández-Zavala $M$, Díaz-García ML, Murata F. Nivel de cumplimiento de la Norma Oficial Mexicana 168-SSA1-1998 del expediente clínico por el personal de enfermería de un Instituto Nacional de Salud. Rev CONAMED 2010; 15 supl. 1:17-22

22 Hernández CS, Hernández ML, Pérez BL, Retiz OA, Vázquez SL, Uribe BB. Repercusión de un programa de mejora continua en los registros de enfermería. Enf Neurol (Mex). 2010; 9(1): 13-6. 